\title{
Dispersion Effects of High-Order-Mode Fiber on Temperature and Axial Strain Discrimination
}

\author{
Yanping $\mathrm{XU}^{1}$, Ping $\mathrm{LU}^{1 *}$, Jia $\mathrm{SONG}^{1}$, Ping $\mathrm{LU}^{2}$, Liang $\mathrm{CHEN}^{1}$, Xiaoyi BAO ${ }^{1^{* *}}$, \\ and Xiaopeng $\mathrm{DONG}^{3}$ \\ ${ }^{1}$ Department of Physics, University of Ottawa, Ottawa, Ontario K1N 6N5, Canada \\ ${ }^{2}$ National Research Council Canada, Ottawa, ON K1A 0R6, Canada \\ ${ }^{3}$ Institute of Lightwave Technology, School of Information Science and Technology, Xiamen University, Xiamen, 361005, \\ China \\ *Corresponding author: Ping LU \\ E-mail: plu@uottawa.ca \\ ${ }^{* *}$ Corresponding author: Xiaoyi BAO \\ E-mail:xbao@uottawa.ca
}

\begin{abstract}
A new approach utilizing effects of dispersion in the high-order-mode fibers (HOMFs) to effectively discriminate changes in environmental temperature and axial strain is proposed and experimentally demonstrated. Experimental characterization of a HOMF-based fiber modal interferometer with a sandwich fiber structure exhibits excellent agreements with numerical simulation results. A Fourier transform method of interferometry in the spatial frequency domain is adopted to distinguish mode coupling between different core-guided modes. Distinct phase sensitivities of multiple dispersion peaks are extracted by employing a novel phase demodulation scheme to realize dual-parameter sensing.
\end{abstract}

Keywords: Optical fiber sensor, temperature and strain discrimination, dispersion

Citation: Yanping XU, Ping LU, Jia SONG, Ping LU, Liang CHEN, Xiaoyi BAO, and Xiaopeng DONG, "Dispersion Effects of High-Order-Mode Fiber on Temperature and Axial Strain Discrimination,” Photonic Sensors, 2015, 5(3): 224-234.

\section{Introduction}

In-situ monitoring of temperature and strain is of great importance for many applications such as biomedical engineering, non-destructive evaluation of civil infrastructure, and environmental monitoring including earthquake and volcano surveillance. A wide variety of optical fiber based sensing techniques have been proposed to meet the requirement of simultaneous measurement of these two important parameters owing to their unique advantages such as immunity to electromagnetic interference, compact size, potential low cost, and the possibility of distributed measurement over a long distance [1-14]. Recently, high-order-mode fibers (HOMFs) have been widely investigated in many applications, such as dispersion compensation, dispersion control of slow-light propagation, generation of Brillouin dynamic gratings, and transverse mode switchable fiber lasers [15-18]. One of the most significant properties of HOMFs is several higher-order core-guided modes are deliberately introduced which exhibit strong chromatic dispersion, thus characterization of HOMFs is vital to greatly facilitate the design of complex fiber geometry and enhance performance of HOMF-based devices. Various dispersion

Received version: 3 March 2015 / Revised version: 22 May 2015

(C) The Author(s) 2015. This article is published with open access at Springerlink.com

DOI: $10.1007 / \mathrm{s} 13320-015-0249-9$

Article type: Regualr 
measurement techniques for HOMFs have been developed, including the white-light interferometric technique [19], optical frequency modulated continuous-wave interferometry [20], phasesensitive optical low coherence reflectometry [21], and time-of-flight method [22]. In this paper, a HOMF-based fiber modal interferometer supporting three dominant modes is achieved with distinct chromatic dispersion of each guided mode and intermodal dispersion. Compared to conventional multimode interference structures (SMF-multimodeSMF structures, taper or lateral-offset in fiber Mach-Zehnder interferometers [23-35]) with a great amount of high-order optical modes, the proposed HOMF modal interferometer supports only a few steady core-guided modes which only need to be calibrated once for sensing response which is essential for practical applications. Thus the HOMF-based sensor here could be deployed for highly accurate discrimination based on the precise dispersion measurement and analysis on intermodal coupling with a novel phase demodulation scheme. It also exhibits distinct temperature and axial strain responses due to the unique wavelength dependent waveguide properties, provides high sensitivities to changes in environmental variables, and possesses a potential to be utilized in multi-parameter measurement scenarios.

\section{Operation principles}

Figure 1 presents a refractive index profile of a custom designed HOMF measured by an optical fiber analyzer (NR-9200, EXFO) based on the refracted near-field technique. Scanning electron microscopy (SEM) images of the symmetrical transversal cross-section of the HOMF are shown in the inset of Fig. 1. The HOMF is etched with $5 \%$ hydrofluoric acid solution for 5 minutes to enhance contrast and visibility of the SEM images because of different etch rates of fiber regions with varied dopant concentrations. The cladding region consists of pure silica with an index of refraction measured to be $1.4560 \pm 0.0001$ at $670 \mathrm{~nm}$. The complex fiber core structure consists of four distinct layers where the central layer is further divided into three subsections. The unique refractive index profile of the HOMF is capable of supporting multiple core modes with tailored dispersion properties. Two single-mode fibers (SMF-28, Corning) are fusion spliced on both sides of the HOMF to form a SMF-HOMF-SMF structure. When a fundamental Gaussian mode is incident from the input SMF to the sandwiched HOMF, a few guided core modes will be excited due to mode field mismatch. Provided that the SMF and the HOMF are perfectly aligned, only circularly symmetric modes are preferably supported in the HOMF due to the circular symmetry of the input optical mode field in the SMF. Interference between these modes shows a sensitive output spectral response of the HOMF modal interferometer to the influences of environmental parameters such as temperature and strain.

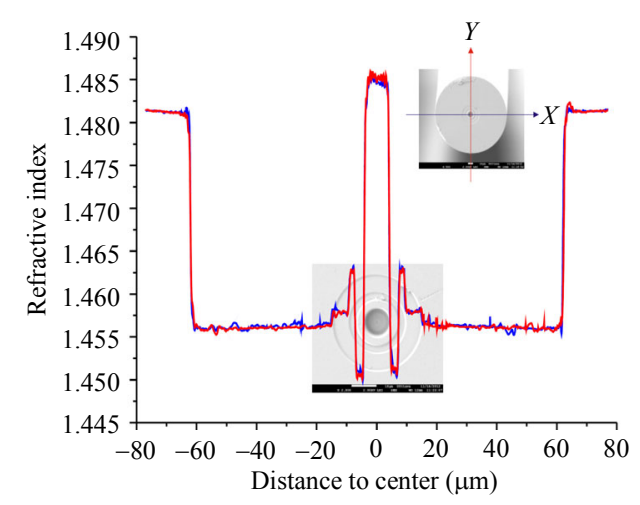

Fig. 1 A calibrated refractive index profile of the HOMF at $670 \mathrm{~nm}$ (two curves correspond to scans along two orthogonal axes of its symmetrical transversal cross-section, respectively; inset: SEM images of the etched HOMF over the whole cross-sectional area and the central core region).

Modal analysis in the HOMF is achieved by employing the COMSOL multiphysics finite element analysis software to numerically simulate the effective indices of the multiple core modes as a function of the wavelength, from which intermodal dispersion and chromatic dispersion properties can be derived. The simulated mode field patterns of the $\mathrm{LP}_{\mathrm{SMF}}$ mode of the SMF as well as the $m$ th order LP $_{0 m}$ modes $(m=1,2,3)$ of the HOMF at $1550 \mathrm{~nm}$ 
are shown in the insets of Figs.2(a) and 2(b). The vector overlap integrals of the two mode fields are performed using the equation [36]:

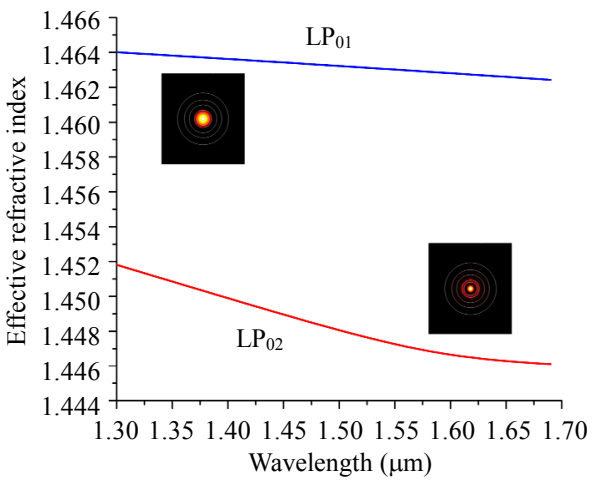

(a)

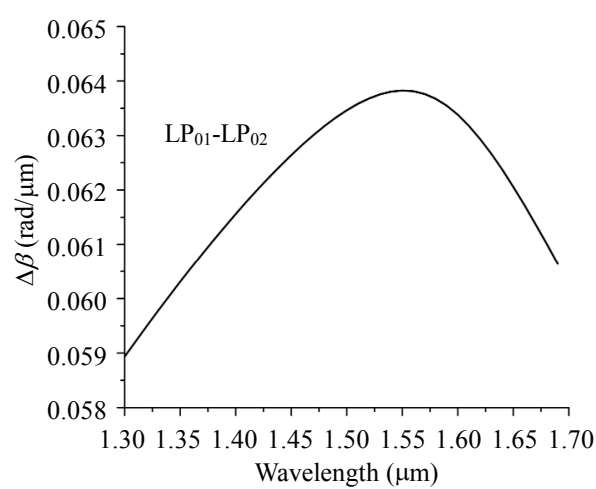

(c)

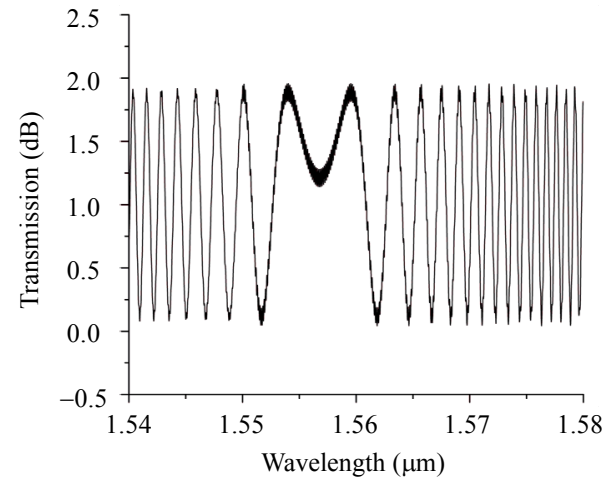

(e)

$$
\eta=\frac{\left|\iint E_{\mathrm{SMF}}(x, y) E_{0 m}^{*}(x, y) d x d y\right|^{2}}{\iint E_{\mathrm{SMF}} E_{\mathrm{SMF}}^{*} d x d y \iint E_{0 m} E_{0 m}^{*} d x d y}
$$

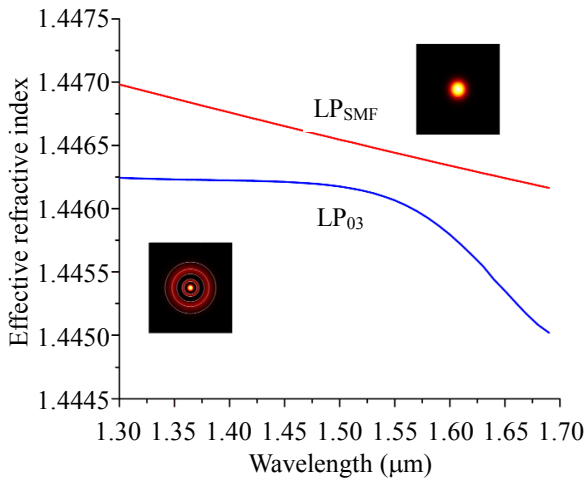

(b)

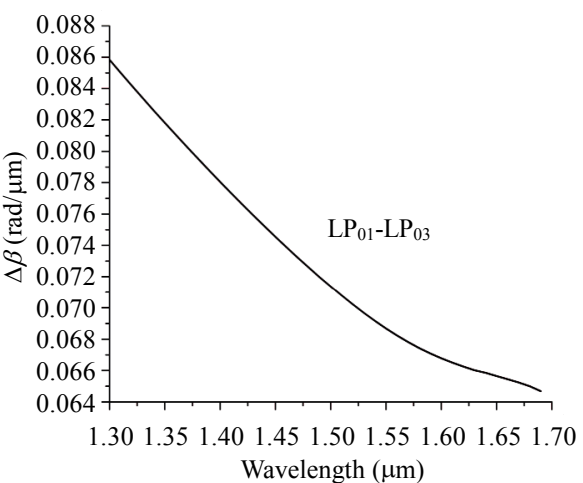

(d)

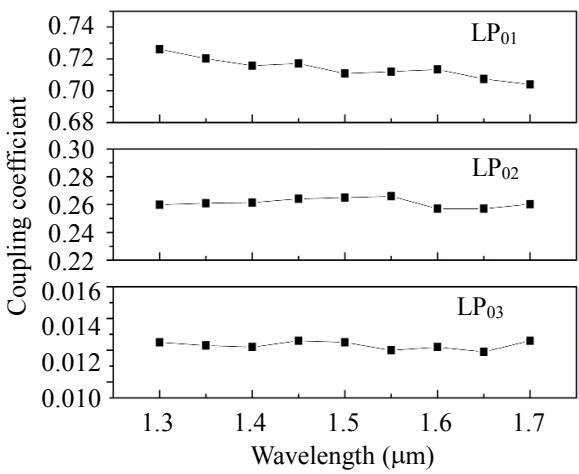

(f)

Fig. 2 Wavelength dependent effective refractive indices of (a) the $\mathrm{LP}_{01}$ and $\mathrm{LP}_{02}$ modes, (b) the $\mathrm{LP}_{03}$ and LPSMF modes; difference in the propagation constant as a function of the wavelength between the $\mathrm{LP}_{01}$ mode and (c) the $\mathrm{LP}_{02}$ mode, $(\mathrm{d})$ the $\mathrm{LP}_{03}$ mode (insets of (a) and (b) show the mode field patterns of the $\mathrm{LP}_{01}$ and $\mathrm{LP}_{02}$ modes, and the $\mathrm{LP}_{03}$ and LPSMF modes, respectively); (e) transmission spectrum of the 1-meter HOMF modal interferometer with three-mode coupling; (f) the coupling coefficients of $\mathrm{LP}_{01}$, $\mathrm{LP}_{02}$, and $\mathrm{LP}_{03}$ at wavelengths ranging from $1.3 \mu \mathrm{m}$ to $1.7 \mu \mathrm{m}$, respectively. 
where $E_{\mathrm{SMF}}$ and $E_{0 m}$ are the complex electric fields of the fundamental mode in the SMF and individual excited core modes in the HOMF, respectively. Coupling efficiencies between the $\mathrm{LP}_{\mathrm{SMF}}$ mode and the $\mathrm{LP}_{01}, \mathrm{LP}_{02}$, and $\mathrm{LP}_{03}$ modes are calculated at different wavelengths in the range of $1.3 \mu \mathrm{m}-$ $1.7 \mu \mathrm{m}$ as shown in Fig. 2(f). It is found that the coupling coefficients for the three core modes maintain quite stable values over the given range with less than $3 \%$ fluctuations, and typical values at $1550 \mathrm{~nm}$ are $71.2 \%, 26.6 \%$, and $1.2 \%$, respectively. Strong coupling between the dominant $\mathrm{LP}_{01}$ mode and the $\mathrm{LP}_{02}$ and $\mathrm{LP}_{03}$ modes exists over a wide range of the wavelength considering their large field overlaps. Since the first three excited core modes in the HOMF account for $99.0 \%$ of the incident light energy, coupling between the $\mathrm{LP}_{01}$ mode and higher-order core modes $(m>3)$ as well as the $\mathrm{LP}_{02}-\mathrm{LP}_{03}$ mode coupling could be negligible.

The wavelength dependent effective indices of the first three core modes in the HOMF are shown in Figs. 2(a) and 2(b). The negative slopes of these three curves follow the same trend of change in the wavelength dependent effective index of the fundamental mode in the SMF as shown in Fig. 2(b). The difference in the propagation constant between two coupling modes is given by

$$
\begin{aligned}
& \Delta \beta_{i j}=\beta_{i}-\beta_{j}=k\left[n_{i}(\lambda)-n_{j}(\lambda)\right]=k \Delta n_{i j}(\lambda) \\
& (i, j=1,2 \text { or } 1,3 \text { or } 2,3)
\end{aligned}
$$

where $k=2 \pi / \lambda$ is the wavenumber, $n_{i(j)}(\lambda)$ and $\Delta n_{i j}(\lambda)$ are the wavelength dependent modal effective indices of the three core modes and their effective index difference, respectively. The intensity in the interference pattern can be written as a form of three-mode coupling:

$$
\begin{aligned}
& I=I_{1}+I_{2}+I_{3}+2 \sqrt{I_{1} I_{2}} \cos \left(\Delta \Phi_{12}(\lambda)\right) \\
& +2 \sqrt{I_{1} I_{3}} \cos \left(\Delta \Phi_{13}(\lambda)\right)+2 \sqrt{I_{2} I_{3}} \cos \left(\Delta \Phi_{23}(\lambda)\right)
\end{aligned}
$$

where $I_{1}, I_{2}$, and $I_{3}$ are the respective intensities of the first-order, the second-order, and third-order core modes, and $\Delta \Phi_{i j}(\lambda)$ is the phase difference between the three core modes with an expression of $\Delta \Phi_{i j}(\lambda)=$
$\Delta \beta_{i j} L$, where $L$ is the length of the HOMF. An approximation is made by neglecting the third interfering term in (3) due to small values of $I_{2}$ and $I_{3}$ compared with $I_{1}$. Figure 2(c) shows the propagation constant difference between the $\mathrm{LP}_{01}$ and the $\mathrm{LP}_{02}$ modes as a function of the wavelength, where a vertex orients upwards existing in the parabolic curve at around $1560 \mathrm{~nm}$ that corresponds to a critical wavelength in the transmission spectrum induced by the $\mathrm{LP}_{01}-\mathrm{LP}_{02}$ mode interference. Figure 2(d) shows the propagation constant difference between the $\mathrm{LP}_{01}$ and the $\mathrm{LP}_{03}$ modes as a function of the wavelength, where no turning point is found and only a monotonically declining curve is obtained within the same wavelength range from $1.3 \mu \mathrm{m}$ to $1.7 \mu \mathrm{m}$. A normalized transmission spectrum of the three-mode coupling interferometer with a 1-meter HOMF can be calculated from (3) by applying a polynomial curve fitting to the data in Figs. 2(c) and 2(d), and the simulation result is shown in Fig. 2(e).

\section{Experimental results and discussion}

Figure 3(a) shows a transmission spectrum of the 1-m HOMF modal interferometer obtained by launching light from a broadband light source to an optical spectrum analyzer (86142A, Agilent). Modal behaviors of the $\mathrm{LP}_{01}-\mathrm{LP}_{02}$ modes allow a high-visibility interference pattern where the critical wavelength locates at $1559.4 \mathrm{~nm}$ that agrees well with the simulation result shown in Fig. 2(e). Furthermore, the inset of Fig. 3(a) shows that there exists a noticeable amplitude modulation in a low-contrast interference fringe due to the presence of the less intense $\mathrm{LP}_{01}-\mathrm{LP}_{03}$ mode coupling. To further investigate HOMF dispersion characteristics, a power spectrum in the spatial frequency domain is obtained and shown in Fig. 3(b) by performing a Fourier transform on the transmission spectrum in the wavelength domain in Fig. 3(a). The spatial frequency is given by [26]

$$
\xi_{2(3)}(\lambda)=1 / \Delta \lambda \approx n_{2(3)}(\lambda) L / \lambda_{0}^{2}
$$


where $\Delta \lambda$ is the wavelength spacing between two neighboring peaks of the interference spectrum, and $\lambda_{0}$ is the central peak wavelength around which a Taylor series is expanded. Figure 3(b) and its inset exhibit two separate clusters of multiple peaks that correspond to two classes of core mode coupling with significant chromatic dispersion effects within the recorded wavelength range from $1540 \mathrm{~nm}$ to $1580 \mathrm{~nm}$. One dominant group of chromatic peaks ranges from $0.2 \mathrm{~nm}^{-1}-3 \mathrm{~nm}^{-1}$ that corresponds to the $\mathrm{LP}_{01}-\mathrm{LP}_{02}$ mode coupling. The lower spatial

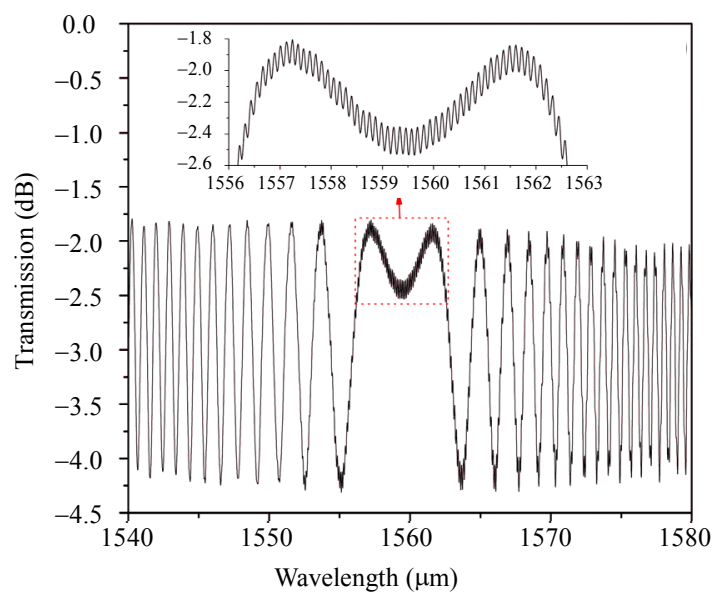

(a) frequency limit of $0.2 \mathrm{~nm}^{-1}$ is determined by the largest peak separation which is about $5 \mathrm{~nm}$ near the critical wavelength in the transmission spectrum. Spatial frequency peaks locating below $0.2 \mathrm{~nm}^{-1}$ correspond to the low frequency modulation in the spectral envelope due to the fluctuation of the laser output, low frequency vibration noises, and other external disturbances. The other group of chromatic peaks extends a spatial frequency range from $6.5 \mathrm{~nm}^{-1}-9.5 \mathrm{~nm}^{-1}$, corresponding to the $\mathrm{LP}_{01}-\mathrm{LP}_{03}$ mode coupling.

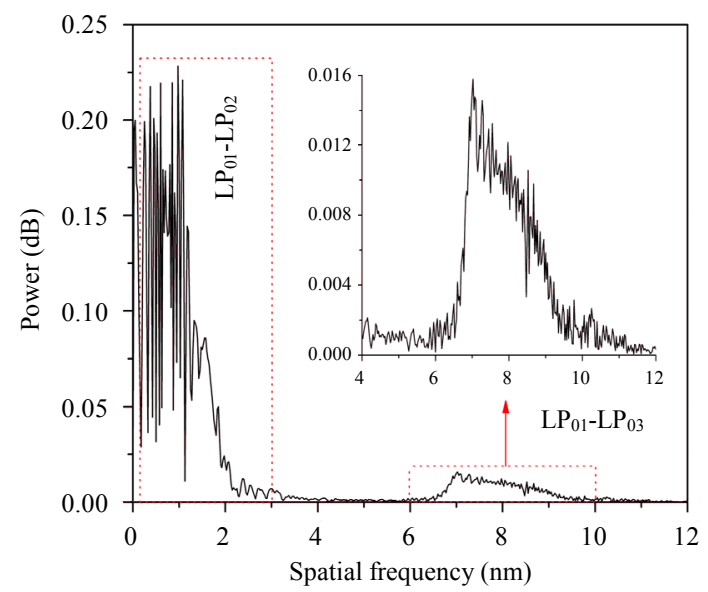

(b)

Fig. 3 1-meter HOMF modal interferometer: (a) transmission spectrum (inset: an enlargement around the critical wavelength of $1559.4 \mathrm{~nm}$ ) and (b) power spectrum in the spatial frequency domain (inset: the group of peaks corresponding to the $\mathrm{LP}_{01}-\mathrm{LP}_{03}$ mode coupling).

Another four HOMF modal interferometers with different HOMF lengths ranging from $75 \mathrm{~cm}$ to $10 \mathrm{~cm}$ are fabricated. Their respective transmission spectra are shown in Figs.4(a), 4(b), and 4(c), and Fig. 4(d) shows the spatial frequencies of two clusters of multiple dispersion peaks in the corresponding power spectra in the spatial frequency domain as a function of the HOMF length. It is indicated that a longer interferometer (HOMF) length may cover a wider range of higher spatial frequencies of multiple dispersion peaks. Provided that more dispersion information is available, higher response to multiple measurands could be potentially obtained and utilized to reduce the effects of cross-talk. The fiber modal interferometer can discriminate axial strain and temperature effects in a more compact way as the length of the HOMF is further shortened down to $1 \mathrm{~cm}$ in the SMF-HOMF-SMF structure. Meanwhile, a shorter length of the HOMF creates less spatial modes, higher degree of material homogeneity and structural regularity which leads to a higher level of measurement accuracy.

Since the peak wavelength spacing is inversely proportional to the length of the HOMF, the amplitude modulation in the interference fringes of the 1-cm HOMF modal interferometer as shown in the bottom subplot of Fig. 5(a) is due to the $\mathrm{LP}_{01}-\mathrm{LP}_{03}$ mode coupling while the interference fringe envelope is caused by the $\mathrm{LP}_{01}-\mathrm{LP}_{02}$ mode 
coupling. The simulated transmission spectrum as shown in the top subplot of Fig. 5(a) provides mode coupling fringes as well as a general envelope closely analogous to the experimental one, verifying the existence of three co-propagating core modes inside the HOMF. Figure 5(b) shows the power spectrum of the 1-cm HOMF modal interferometer in the spatial frequency domain, where a dominant peak for the $\mathrm{LP}_{02}$ mode locates at $0.0222 \mathrm{~nm}^{-1}$, and three other lower peaks for the $\operatorname{LP}_{03-i}(i=1,2,3)$ locate at $0.0444 \mathrm{~nm}^{-1}, 0.0888 \mathrm{~nm}^{-1}$, and $0.1222 \mathrm{~nm}^{-1}$, respectively, corresponding to wavelength dependent effective indices of the $\mathrm{LP}_{01}-\mathrm{LP}_{03}$ mode coupling. The spatial frequency range from $0.0444 \mathrm{~nm}^{-1}$ to $0.1222 \mathrm{~nm}^{-1}$ corresponds to a range of $8 \mathrm{~nm}$ to $22.5 \mathrm{~nm}$ in the wavelength spacing. The

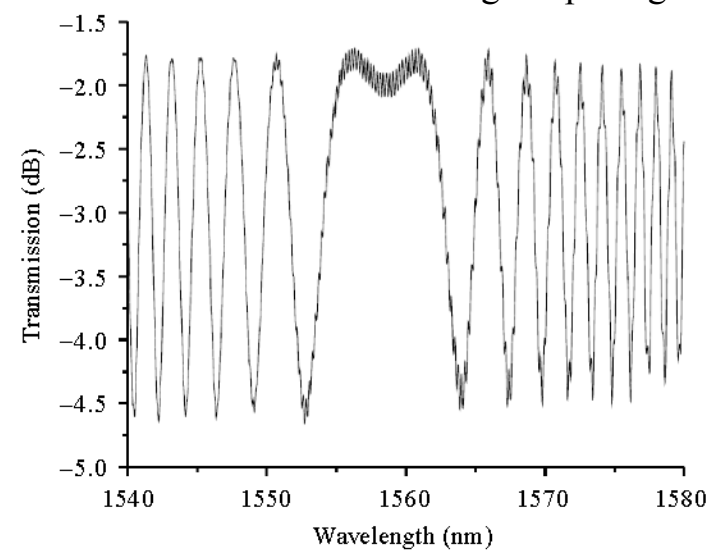

(b)

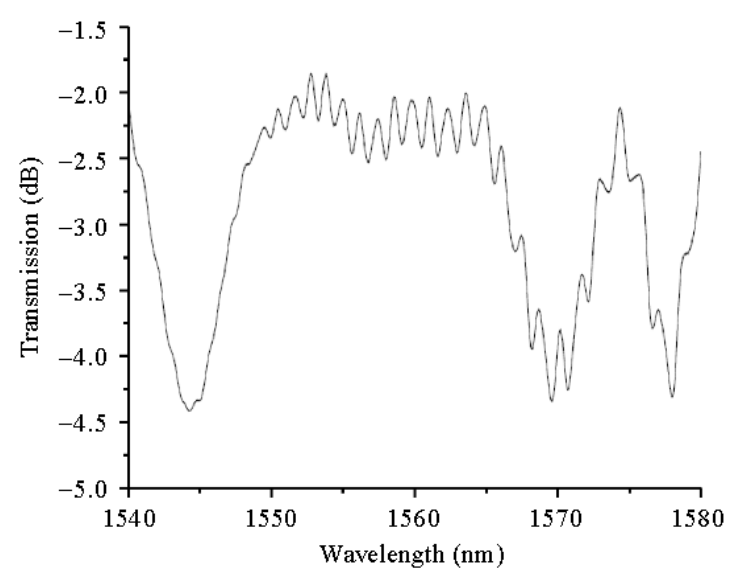

actual maximum wavelength spacing in the experimental transmission spectrum as shown in Fig. 5(a) is $17 \mathrm{~nm}$ that is obtained from the two wavelength peaks located at around $1570 \mathrm{~nm}$ and $1587 \mathrm{~nm}$. This discrepancy is generated as a result of the limited resolution in the spatial frequency domain because of the insufficient data points to calculate the Fourier transform. Compared with the 1-m HOMF modal interferometer, less dispersion information is carried in the 1-cm one even for a larger wavelength span since the spatial frequency is proportional to the HOMF length. Figures5(c) and 5(d) show a series of phase spectra in the spatial frequency domain at different temperatures and strains where the normalized phase between 0 and $2 \pi$ are obtained from Fourier transformed data at each spatial frequency, respectively.

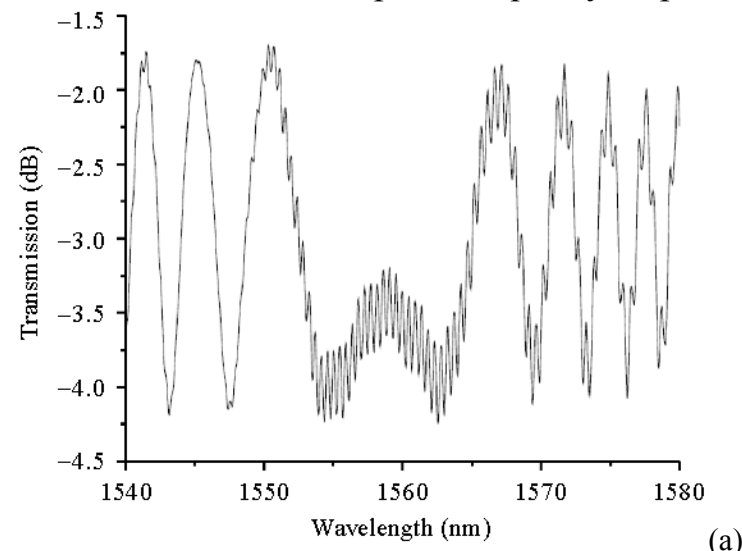

(a)

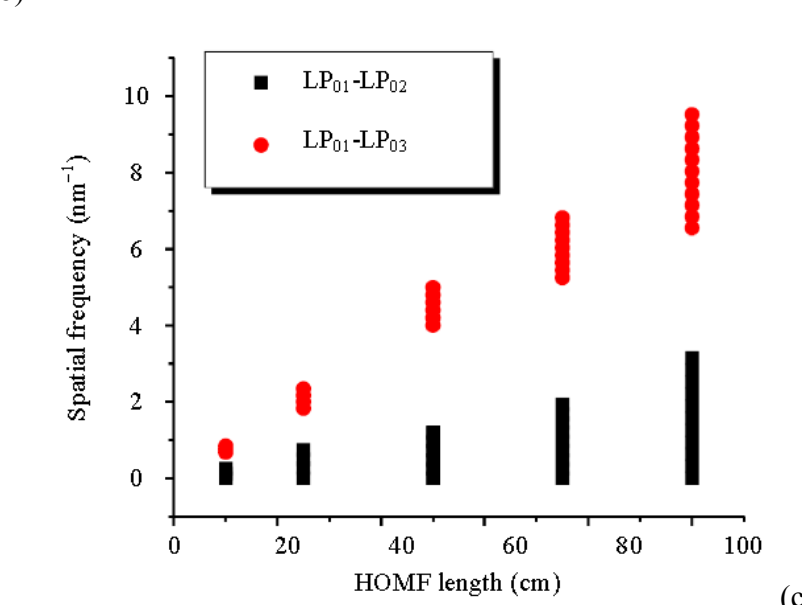

(d)

Fig. 4 Transmission spectra of the HOMF modal interferometer with a HOMF length of (a) $50 \mathrm{~cm}$, (b) $25 \mathrm{~cm}$, and (c) $10 \mathrm{~cm}$, respectively; (d) dependence of the spatial frequencies of two clusters of multiple dispersion peaks on the HOMF length. 


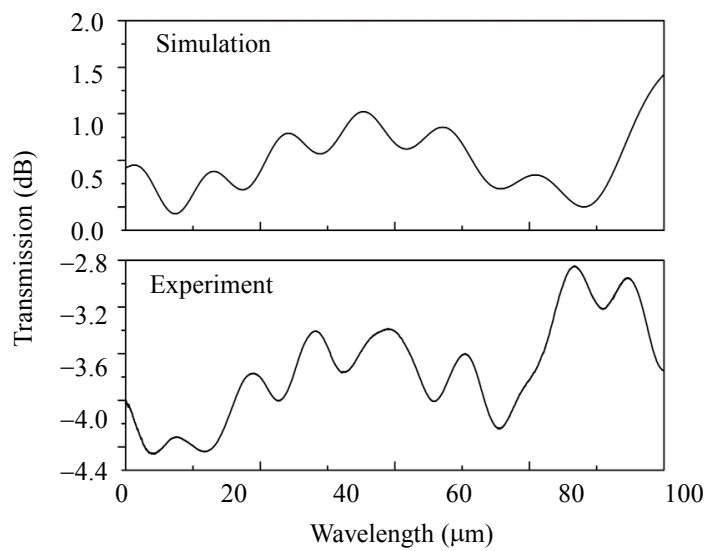

(a)

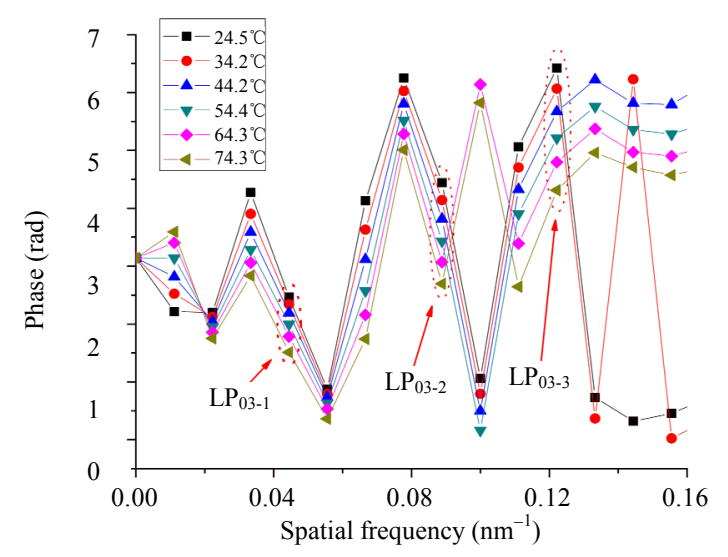

(c)

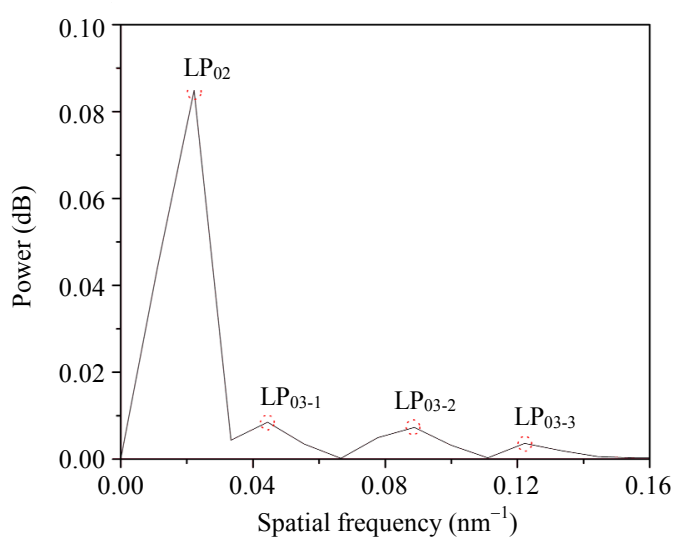

(b)

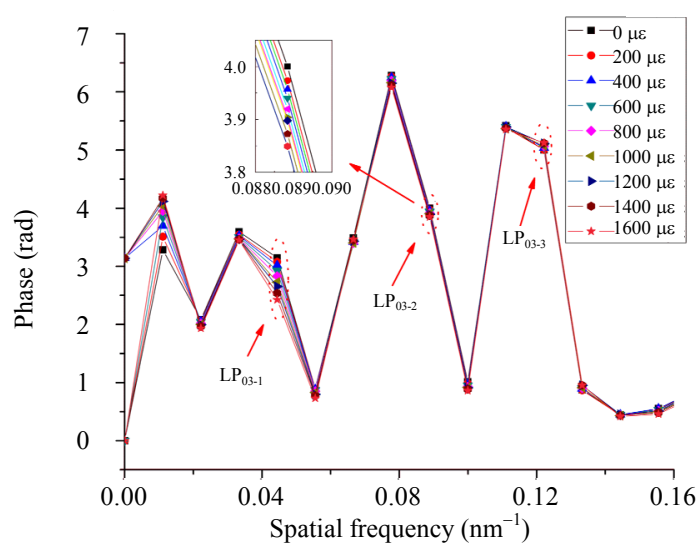

(d)

Fig. 5 1-centimeter HOMF modal interferometer: (a) top: simulated transmission spectrum; bottom: experimental transmission spectrum; (b) experimental power spectrum in the spatial frequency domain; experimental phase spectra in the spatial frequency domain (c) at different temperatures and (d) at different axial strains.

The shift in the phase difference between the $\mathrm{LP}_{01}$ mode and the $\mathrm{LP}_{02}$ or $\mathrm{LP}_{03}$ mode at a particular spatial frequency can be expressed by $\delta\left(\Delta \Phi_{T(\varepsilon)}\right)=-$ $2 \pi \xi \delta \lambda_{T(\varepsilon)}$ [37], where $\delta \lambda_{T(\varepsilon)}$ is the temperature or axial strain induced peak wavelength shift in the interference spectrum between the $\mathrm{LP}_{01}$ mode and the $\mathrm{LP}_{02}$ or $\mathrm{LP}_{03}$ mode. In Fig. 5(b), the peak with the highest intensity corresponds to the $\mathrm{LP}_{01}-\mathrm{LP}_{02}$ mode coupling in a large spectral range, which may exhibit cross-talk on chromatic dispersion and poor linearity under changes in temperature and axial strain. Thus the group of three peaks originating from strong dispersion effects of the $\mathrm{LP}_{01}-\mathrm{LP}_{03}$ mode coupling is utilized for temperature and axial strain discrimination because of their different responses in modal effective indices under equal external disturbance conditions. For the axial strain and temperature measurement with the 1-cm HOMF modal interferometer, the optical fiber is fixed on two linear motion stages with a translation accuracy of $1 \mu \mathrm{m}$ using epoxy glue and axially stretched at different temperatures controlled by an oven with the temperature accuracy of $0.1{ }^{\circ} \mathrm{C}$. The temperature increases with a step of $10{ }^{\circ} \mathrm{C}$ from an initial temperature of $24{ }^{\circ} \mathrm{C}$ with a constant strain applied on the fiber to calibrate the temperature response, and an axial strain of $1600 \mu \varepsilon$ with a step of $200 \mu \varepsilon$ is applied on the fiber with a constant temperature of 
$24{ }^{\circ} \mathrm{C}$ to calibrate the axial strain response. It is noted that the phase shift is negatively proportional to the corresponding interference peak wavelength shift which is given by

$$
\delta \lambda_{T(\varepsilon)}=\left(\frac{\delta L_{T(\varepsilon)}}{L}+\frac{\delta\left(\Delta n_{T(\varepsilon)}\right)}{\Delta n_{T(\varepsilon)}}\right) \lambda
$$

where $\delta L_{T(\varepsilon)}$ and $\delta\left(\Delta n_{T(\varepsilon)}\right)$ are changes in the length of the HOMF and the difference in the modal effective indices. Both the fiber length and the difference in the modal effective indices will increase due to the thermo-elastic and thermo-optic effects with an increasing temperature. Therefore, the phase difference of the HOMF modal interferometer will always experience blueshift. When the axial strain is applied on the HOMF modal interferometer, the fiber will be stretched, and the difference in the modal effective indices is contributed by both the fiber geometry modification and the photo-elastic effect which makes the

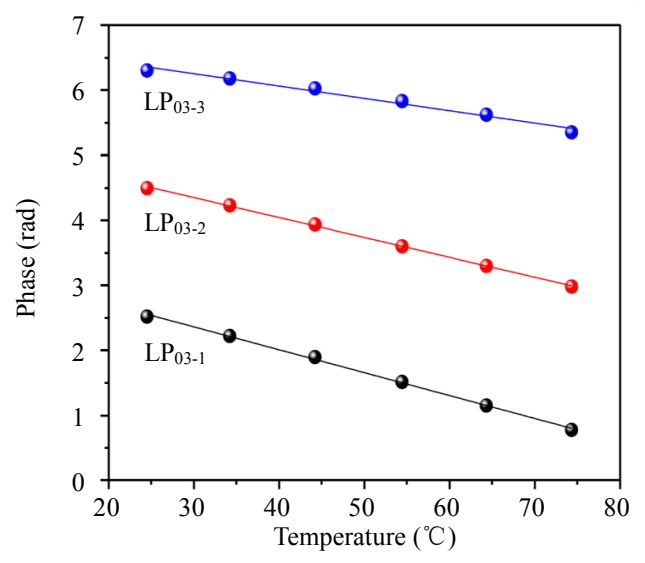

(a) positive and negative signs of $\delta\left(\Delta n_{\varepsilon}\right)$ both possible. Thus the phase difference will experience either blueshift or redshift, depending upon the relative magnitudes of $\delta L_{\varepsilon} / L_{\varepsilon}$ and $\delta\left(\Delta n_{\varepsilon}\right) / \Delta n_{\varepsilon}$. Figure 6 shows experimental results of temperature and axial strain dependences of the phase difference at different spatial frequencies corresponding to distinct chromatic dispersion of the $\mathrm{LP}_{01}-\mathrm{LP}_{03}$ modes. As shown in Fig. 6(a), temperature coefficients of the $\mathrm{LP}_{03-1}, \quad \mathrm{LP}_{03-2}$, and $\mathrm{LP}_{03-3}$ peaks are $-0.0353 \pm 0.0007 \mathrm{rad} /{ }^{\circ} \mathrm{C},-0.0307 \pm 0.0004 \mathrm{rad} /{ }^{\circ} \mathrm{C}$, and $-0.019 \pm 0.001 \mathrm{rad} /{ }^{\circ} \mathrm{C}$, respectively. As shown in Fig. 6(b), the axial strain coefficients of the $\mathrm{LP}_{03-1}, \quad \mathrm{LP}_{03-2}$, and $\mathrm{LP}_{03-3}$ peaks are $-454 \pm 14 \mathrm{rad} / \varepsilon, \quad-88 \pm 3 \mathrm{rad} / \varepsilon$, and $85 \pm 6 \mathrm{rad} / \varepsilon$, respectively. Different peaks have distinctive temperature and axial strain sensitivities that effectively enables simultaneous measurement of multi-parameters.

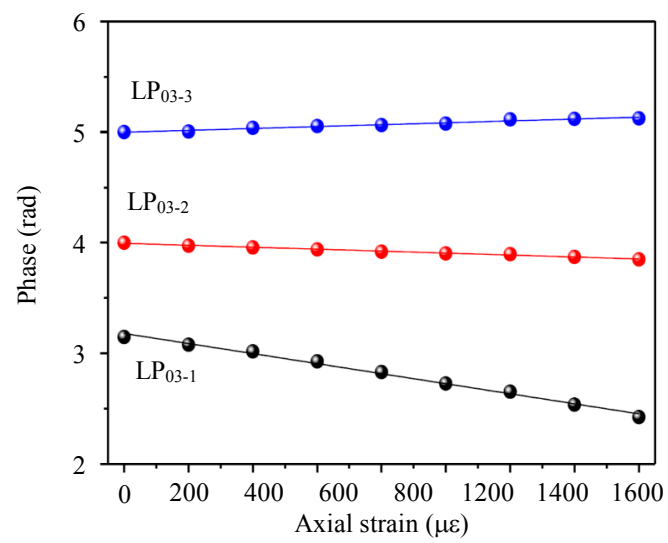

(b)

Fig. 6 Experimental results of phase versus (a) temperature and (b) axial strain at different spatial frequencies.

A character matrix $M_{T, \varepsilon}^{m}$ is defined to represent the sensing performance of the $m$ th order $\mathrm{LP}_{0 m}$ modes in the HOMF modal interferometer by

$$
\left[\begin{array}{l}
\delta \Phi_{0 m-i} \\
\delta \Phi_{0 m-i^{-}}
\end{array}\right]=M_{T, \varepsilon}^{m}\left[\begin{array}{l}
\Delta T \\
\Delta \varepsilon
\end{array}\right]=\left[\begin{array}{ll}
C_{0 m-i}^{T} & C_{0 m-i}^{\varepsilon} \\
C_{0 m-i^{i}}^{T} & C_{0 m-i^{-}}^{\varepsilon}
\end{array}\right]\left[\begin{array}{c}
\Delta T \\
\Delta \varepsilon
\end{array}\right]
$$

where $i$ and $i^{\prime}$ represent two arbitrary selected $\mathrm{LP}_{0 m-i}$ peaks for the temperature and axial strain discrimination. $C_{03-i}{ }^{T}, C_{03-i^{i}}{ }^{T}, C_{03-i}{ }^{\varepsilon}$, and $C_{03-i}{ }^{\varepsilon}$ are the temperature and axial strain coefficients of the $\mathrm{LP}_{03-i}$ and $\mathrm{LP}_{03-i^{\prime}}$ peaks, respectively. The error analysis for the simultaneous measurement of multiple parameters is given by [38]

$$
\begin{gathered}
\delta T=\frac{\left|C_{03-i}^{\varepsilon}\right| \delta \Phi_{03-i}+\left|C_{03-i}^{\varepsilon}\right| \delta \Phi_{03-i}}{\left|C_{03-i}^{T} C_{03-i^{i}}^{\varepsilon}-C_{03-i}^{\varepsilon} C_{03-i^{2}}^{T}\right|}, \\
\delta \varepsilon=\frac{\left|C_{03-i}^{T}\right| \delta \Phi_{03-i}+\left|C_{03-i^{i}}^{T}\right| \delta \Phi_{03-i^{\prime}}}{\left|C_{03-i}^{T} C_{03-i^{i}}^{\varepsilon}-C_{03-i}^{\varepsilon} C_{03-i^{2}}^{T}\right|} .
\end{gathered}
$$


The minimum errors of temperature and axial strain are calculated to be $0.15^{\circ} \mathrm{C}$ and $22 \mu \varepsilon, 0.15^{\circ} \mathrm{C}$ and $19 \mu \varepsilon$, and $0.24{ }^{\circ} \mathrm{C}$ and $68 \mu \varepsilon$, for the mode coupling between $\mathrm{LP}_{01}$ and $\mathrm{LP}_{03-1}, \mathrm{LP}_{03-2}$, and $\mathrm{LP}_{03-3}$, respectively.

\section{Conclusions}

In conclusion, a novel approach to fulfill simultaneous dual-parameter measurement based on the joint effect of chromatic dispersion and intermodal dispersion of a HOMF modal interferometer is proposed and demonstrated. A phase demodulation scheme based on a fast Fourier transform algorithm is innovatively adopted to analyze the mode coupling in the SMF-HOMF-SMF structure and distinguish the excited modes in the dispersion tailored HOMF. The strong dispersion effects of a few core modes lead to the multiple-peak feature in the spatial frequency domain, which provides the availability of abundant choices of sensitivities to different environmental variables. Cross-talk could be further suppressed by introducing more dispersion peaks with higher spatial frequency resolution among which proper selections should be made to maximize the measurand coefficients and enhance discrimination between measurands. By selecting the appropriate length of the central HOMF according to varied actual requirements, the proposed easy-fabricating and compact sensing structure would be potentially applicable in situations where more than two parameters are required to be discriminated.

\section{Acknowledgment}

The research is supported by the Natural Sciences and Engineering Research Council of Canada (NSERC) Discovery Grants and Canada Research Chairs (CRC) Program. Ping Lu would like to acknowledge the Province of Ontario Ministry of Research and Innovation and the University of Ottawa for the financial support of the
Vision 2020 Postdoctoral Fellowship.

Open Access This article is distributed under the terms of the Creative Commons Attribution License which permits any use, distribution, and reproduction in any medium, provided the original author(s) and source are credited.

\section{References}

[1] H. F. Lima, P. F. Antunes, J. D. L. Pinto, and R. N. Nogueira, "Simultaneous measurement of strain and temperature with a single fiber Bragg grating written in a tapered optical fiber," IEEE Sensors Journal, 2010, 10(2): 269-273.

[2] P. Lu and Q. Chen, "Asymmetrical fiber Mach-Zehnder interferometer for simultaneous measurement of axial strain and temperature," IEEE Photonics Journal, 2010, 2(6): 942-953.

[3] Y. N. Tan, Y. Zhang, L. Jin, and B. O. Guan, "Simultaneous strain and temperature fiber grating laser sensor based on radio-frequency measurement," Optics Express, 2011, 19(21): 20650-20656.

[4] L. M. Hu, C. C. Chan, X. Y. Dong, Y. P. Wang, P. Zu, W. C. Wong, et al., "Photonic crystal fiber strain sensor based on modified Mach-Zehnder interferometer," IEEE Photonics Journal, 2012, 4(1): 114-118.

[5] H. R. Alemohammad, E. Foroozmehr, B. S. Cotten, and E. Toyserkani, "A dual-parameter optical fiber sensor for concurrent strain and temperature measurement: design, fabrication, packaging, and calibration," IEEE Journal of Lightwave Technology, 2013, 31(8): 1198-1204.

[6] O. Frazao, J. P. Carvalho, L. A. Ferreira, F. M. Araujo, and J. L. Santos, "Discrimination of strain and temperature using Bragg gratings in microstructured and standard optical fibres," Measurement Science and Technology, 2005, 16(10): 2109-2113.

[7] O. Frazao, L. M. Marques, S. Santos, J. M. Baptista, and J. L. Santos, "Simultaneous measurement for strain and temperature based on a long-period grating combined with a high-birefringence fiber loop mirror," IEEE Photonic Technology Letter, 2006, 18(22): 2407-2409.

[8] O. Frazao and J. L. Santos, "Simultaneous measurement of strain and temperature using a Bragg grating structure written in germanosilicate fibres," Journal of Optics A: Pure and Applied Optics, 2004, 6(6): 553-556.

[9] O. Frazao, R. Romero, F. M. Araujo, L. A. Ferreira, and J. L. Santos, "Strain-temperature discrimination using a step spectrum profile fibre Bragg grating 
arrangement," Sensors and Actuators A: Physical, 2005, 120(2): 490-493.

[10] I. Abe, H. J. Kalinowski, O. Frazao, J. L. Santos, R. N. Nogueira, and J. L. Pinto, "Superimposed Bragg gratings in high-birefringence fibre optics: three-parameter simultaneous measurements," Measurement Science and Technology, 2004, 15(8): 1453-1457.

[11] C. L. Zhao, J. R. Zhao, W. Jin, J. Ju, L. Cheng, and X. G. Huang, "Simultaneous strain and temperature measurement using a highly birefringence fiber loop mirror and a long-period grating written in a photonic crystal fiber," Optics Communications, 2009, 282(20): 4077-4080.

[12] T. Liu, G. F. Fernando, Z. Y. Zhang, and K. T. V. Grattan, "Simultaneous strain and temperature measurements in composites using extrinsic Fabry-Perot interferometric and intrinsic rare-earth doped fiber sensors," Sensors and Actuators A: Physical, 2000, 80(3): 208-215.

[13] K. Kishida, Y. Yamauchi, and A. Guzik, "Study of optical fibers strain-temperature sensitivities using hybrid Brillouin-Rayleigh system," Photonic Sensors, 2014, 4(1): 1-11.

[14] K. Hotate, W. Zou, R. K. Yamashita, and Z. He, "Distributed discrimination of strain and temperature based on Brillouin dynamic grating in an optical fiber," Photonic Sensors, 2013, 3(4): 332-344.

[15] S. Ramachandran, "Dispersion-tailored few-mode fibers: a versatile platform for in-fiber photonic devices," IEEE Journal of Lightwave Technology, 2005, 23(11): 3426-3443.

[16] A. A. Sukhorukov, C. J. Handmer, C. M. de Sterke, and M. J. Steel, "Slow light with flat or offset band edges in few-mode fiber with two gratings," Optics Express, 2007, 15(26): 17954-17959.

[17] S. Li, M. J. Li, and R. S. Vodhanel, "All-optical Brillouin dynamic grating generation in few-mode optical fiber," Optics Letters, 2012, 37(22): 4660-4662.

[18] B. Sun, A. Wang, L. Xu, C. Gu, Y. Zhou, Z. Lin, et al., "Transverse mode switchable fiber laser through wavelength tuning," Optics Letters, 2013, 38(5): 667-669.

[19] D. Menashe, M. Tur, and Y. Danziger, "Interferometric technique for measuring dispersion of high order modes in optical fibres," Electronics Letters, 2001, 37(24): 1439-1440.

[20] T. J. Ahn, Y. Jung, K. Oh, and D. Y. Kim, "Optical frequency-domain chromatic dispersion measurement method for higher-order modes in an optical fiber," Optics Express, 2005, 13(25): 10040-10048.

[21]P. Hamel, Y. Jaouën, R. Gabet, and S. Ramachandran,
"Optical low-coherence reflectometry for complete chromatic dispersion characterization of few-mode fibers," Optics Letters, 2007, 32(9): 1029-1031.

[22] J. Cheng, M. E. V. Pedersen, K. Wang, C. Xu, L. Grüner-Nielsen, and D. Jakobsen, "Time-domain multimode dispersion measurement in a higherorder-mode fiber," Optics Letters, 2012, 37(3): 347-349.

[23] Q. Wang, G. Farrell, and W. Yan, "Investigation on single-mode-multimode-single-mode fiber structure," IEEE Journal of Lightwave Technology, 2008, 26(5): 512-519.

[24] Q. Wu, Y. Semenova, P. Wang, and G. Farrell, "High sensitivity SMS fiber structure based refractometer analysis and experiment," Optics Express, 2011, 19(9): 7937-7944.

[25] M. Kumar, A. Kumar, and S. M. Tripathi, "A comparison of temperature sensing characteristics of SMS structures using step and graded index multimode fibers," Optics Communications, 2014, 312(1): 222-226.

[26] H. Y. Choi, M. J. Kim, and B. H. Lee, "All-fiber Mach-Zehnder type interferometers formed in photonic crystal fiber," Optics Express, 2007, 15(9): 5711-5720.

[27] P. Lu, L. Men, K. Sooley, and Q. Chen, “Tapered fiber Mach-Zehnder interferometer for simultaneous measurement of refractive index and temperature," Applied Physics Letters, 2009, 94(13): 131110-1-131110-3.

[28] S. Zhang, W. Zhang, S. Gao, P. Geng, and X. Xue, "Fiber-optic bending vector sensor based on Mach-Zehnder interferometer exploiting lateral-offset and up-taper," Optics Letters, 2012, 37(21): 4480-4482.

[29] L. C. Li, L. Xia, Z. H. Xie, and D. M. Liu, "All-fiber Mach-Zehnder interferometers for sensing applications," Optics Express, 2012, 20(10): 11109-11120.

[30] Q. Q. Yao, H. Y. Meng, W. Wang, H. C. Xue, R. Xiong, B. Huang, et al., "Simultaneous measurement of refractive index and temperature based on a core-offset Mach-Zehnder interferometer combined with a fiber Bragg grating," Sensors and Actuators A: Physical, 2014, 209(1): 73-77.

[31] K. Ni, T. Li, L. M. Hu, W. W. Qian, Q. Y. Zhang, and S. Z. Jin, "Temperature-independent curvature sensor based on tapered photonic crystal fiber interferometer," Optics Communications, 2012, 285(24): 5148-5150.

[32] Y. P. Xu, P. Lu, Z. G. Qin, J. Harris, F. Baset, P. Lu, et al., "Vibration sensing using a tapered bend-insensitive fiber based Mach-Zehnder 
interferometer," Optics Express, 2013, 21(3): 3031-3042.

[33] L. Yuan, "Recent progress of in-fiber integrated interferometers," Photonic Sensors, 2011, 1(1): 1-5.

[34]C. Yu, X. Chen, Y. Gong, Y. Wu, Y. Rao, and G. Peng, "Simultaneous force and temperature measurement using optical microfiber asymmetrical interferometer," Photonic Sensors, 2014, 4(3): 242-247.

[35] H. Latifi, M. I. Zibaii, S. M. Hosseini, and P. Jorge, "Nonadiabatic tapered optical fiber for biosensor applications," Photonic Sensors, 2012, 2(4): 340-356.
[36] J. Sakai and T. Kimura, "Design of a miniature lens for semiconductor laser to single-mode fiber coupling," IEEE Journal of Quantum Electronics, 1980, 16(10): 1059-1067.

[37] P. Lu, J. Harris, Y. Xu, Y. Lu, L. Chen, and X. Bao, "Simultaneous refractive index and temperature measurements using a tapered bend-resistant fiber interferometer," Optics Letters, 2012, 37(22): 4567-4569.

[38] W. Jin, W. C. Michie, G. Thursby, M. Konstantaki, and B. Culshaw, "Simultaneous measurement of strain and temperature: error analysis," Optical Engineering, 1997, 36(2): 598-609. 